
\title{
R Reserach S Suare \\ Predialysis Serum Lactate Levels Can Predict Dialysis Withdrawal in Type 1 Cardiorenal Syndrome Patients
}

\author{
Heng-Chih Pan \\ Keelung Chang Gung Memorial Hospital \\ Vincent Wu ( $\sim$ q91421028@ntu.edu.tw) \\ National Taiwan University Hospital
}

\section{Research Article}

Keywords: Type 1 cardiorenal syndrome, renal replacement therapy, withdrawal, serum lactate, prognosis, kidney recovery

Posted Date: February 19th, 2021

DOI: https://doi.org/10.21203/rs.3.rs-209086/v1

License: (c) (i) This work is licensed under a Creative Commons Attribution 4.0 International License.

Read Full License 


\section{Predialysis Serum Lactate Levels Can Predict Dialysis Withdrawal}

\section{in Type 1 Cardiorenal Syndrome Patients}

Heng-Chih Pan ${ }^{1,2,3,4}$, Vin-Cent $\mathrm{Wu}^{5,6}$

1. Chang Gung University College of Medicine, Taoyuan, Taiwan

2. Division of Nephrology, Department of Internal Medicine, Keelung Chang Gung Memorial Hospital, Keelung, Taiwan

3. Graduate Institute of Clinical Medicine, College of Medicine, National Taiwan

University, Taipei, Taiwan

4. Community Medicine Research Center, Keelung Chang Gung Memorial Hospital,

Keelung, Taiwan,

5. Division of Nephrology, Department of Internal Medicine, National Taiwan

University Hospital, Taipei, Taiwan

6. NSARF (National Taiwan University Hospital Study Group of ARF), TAIPAI,

(Taiwan Primary Aldosteronism Investigators), and CAKS (Taiwan Consortium for

Acute Kidney Injury and Renal Diseases), Taipei, Taiwan

Short title: Serum lactate and type 1 CRS requiring RRT 
Correspondence and reprint requests should be made to:

Vin-Cent $\mathrm{Wu}, \mathrm{MD}, \mathrm{PhD}$

Room 1419, Clinical Research Building, Department of Internal Medicine

National Taiwan University Hospital

7 Chung-Shan South Road, Taipei, Taiwan 100

Telephone: $+886-2-23562082$

Fax: +886-2-23934176

E-mail: q91421028@ntu.edu.tw 


\section{Abstract}

Type 1 cardiorenal syndrome (CRS) is a complication with grave outcomes, and renal replacement therapy (RRT) is an effective rescue therapy. Serum lactate has been correlated with the risk of mortality in patients with sepsis. However, the association between serum lactate level and the prognosis of type 1 CRS patients requiring RRT is unknown. We prospectively enrolled 500 type 1 CRS patients who received RRT from August 2011 to January 2018. The 90-day mortality rate was 52.8\% and the incidence rate of RRT independence was $34.8 \%$. Lower pre-dialysis lactate was correlated with a higher rate of dialysis withdrawal and lower rate of mortality. A generalized additive model showed that $4.2 \mathrm{mmol} / \mathrm{L}$ was an adequate cut-off value of lactate to predict renal recovery. Taking mortality as a competing risk, Cox proportional hazards analysis indicated that a low lactate level ( $\leqq 4.2 \mathrm{mmol} / \mathrm{L})$ was an independent prognostic factor for the possibility of dialysis withdrawal, as also shown in external validation. The interaction of quick Sequential Organ Failure Assessment score and lactate was associated with dialysis dependence in a disease severitydependent manner. In summary, we identified that pre-dialysis serum lactate level could predict the possibility of dialysis withdrawal in type 1 CRS patients. 
Keywords: Type 1 cardiorenal syndrome, renal replacement therapy, withdrawal,

serum lactate, prognosis, kidney recovery 


\section{Introduction}

Type 1 cardiorenal syndrome (CRS) is characterized by a rapid worsening of cardiac function and marked systemic congestion that absolutely or relatively causes a secondary insult to kidney function ${ }^{1-3}$. The appearance of CRS generally indicates a grave outcome, such as an increased risk of long-term mortality and end-stage renal disease $^{4}$. Emerging evidence has demonstrated a strong correlation between acute kidney injury (AKI) and systemic inflammation in type 1 CRS patients ${ }^{5}$. In addition, even a moderate degree of kidney function impairment has been independently associated with an increased risk of mortality from any cause in patients with heart failure $(\mathrm{HF})^{6}$. Treatment options for type $1 \mathrm{CRS}$ are still limited, and renal replacement therapy (RRT) is regarded to be an effective rescue therapy when refractory HF symptoms persist ${ }^{7}$. However, objective parameters to provide accurate information on disease severity and evaluate the possibility of kidney recovery or survival in type 1 CRS patients are lacking.

Lactic acid is a biomarker of tissue hypoperfusion, and serum lactate has been demonstrated to be a good indicator of the severity of cardiac shock ${ }^{8}$. Unlike other widely used markers in $\mathrm{HF}$, lactate is unique because it reflects the dynamic 
energetic/metabolic status of these patients ${ }^{9}$. Several investigations have indicated that increased serum lactate is a strong predictor of mortality in the setting of septic

patients requiring $\mathrm{RRT}^{10-12}$, however the relationship between serum lactate levels and the prognosis of type 1 CRS is still unknown. In critical care patients with considerable comorbidities, the competing risk of death is especially high and will confound the clinical analysis of dialysis withdrawal.

The aim of this study was to identify whether predialysis serum lactate can be potential prognostic to predict RRT withdrawal, 90-day survival and competing death in type $1 \mathrm{CRS}$ patients who required dialysis for $\mathrm{AKI}$.

\section{Results}

\section{Baseline Characteristics of the Study Population}

We enrolled 500 eligible patients in this study. The overall 90-day mortality rate was $52.8 \%(264 / 500)$. The patients were divided into three groups: mortality group $(\mathrm{n}=$ 264), dialysis dependence group $(n=62)$, and dialysis withdrawal group $(n=174)$, according to whether they died, were dependent on dialysis, or withdrew from dialysis within 90 days after the initiation of dialysis for AKI. The baseline 
demographic data and clinical characteristics are shown in Table 1. Overall, the mean age of the patients was 61.4 years; 360 patients were men $(72.0 \%)$ and 140 were women (28.0\%). Sepsis was the most common cause of AKI (52.6\%). The baseline SCr level of the dialysis withdrawal group was significantly lower than that of the dialysis dependence group but higher than that of the mortality group.

Comparing the three groups, the mortality group had significantly higher Sequential Organ Failure Assessment (SOFA) and quick Sequential Organ Failure Assessment (qSOFA) scores on ICU admission than the other two groups; and the dialysis dependence group had higher age, baseline SCr, frequency of diabetes and history of PTCA with stents than the other two groups. The dialysis withdrawal group was significantly associated with a higher probability of receiving total parenteral nutrition than the other two groups. The prevalence rates of hypertension, coronary artery disease, smoking, dementia, rheumatologic disease, hemiplegia, peptic ulcer disease, and malignancy, and the severity of congestive HF, involved number of vessels treated by percutaneous transluminal coronary angioplasty (PTCA) and coronary artery bypass graft and the urgency of receiving surgery were similar among the three groups. 
In this study, oliguria (66.6\%) was the leading reason for receiving RRT. Before dialysis, the mortality group had significantly lower systolic blood pressure, daily urine output (UO) and Glasgow coma scale, but higher lactate levels, SOFA and qSOFA scores than the other two groups. In addition, the dialysis withdrawal group had significantly higher Hgb levels than the other two groups. Comparisons of the indications for dialysis revealed that the mortality group had a significantly higher rate of receiving RRT due to metabolic acidosis than the other two groups.

Comparisons of baseline characteristics and clinical parameters before dialysis across the outcome groups were expressed as correlation scatterplots with matrices and Seaborn violin plots (Suppl. Figure 1).

\section{Pre-dialysis Lactate Levels Predicted Dialysis Withdrawal in the Type 1 CRS}

\section{Patients Receiving Dialysis}

We used a nonlinear generalized additive model to identify adequate cut-off values of continuous parameters to predict renal recovery (Figure 1). All of the relevant covariates, including baseline characteristics, comorbidities, clinical parameters before dialysis such as qSOFA score, indications for dialysis, plasma lactate levels and other laboratory data listed in Table 1 were the selected variables. In our cohort, 
we found that lower pre-dialysis lactate (cut-off value: $4.2 \mathrm{mmol} / \mathrm{L}$ ) was associated

with a higher possibility of dialysis withdrawal. We further divided the patients

according to their pre-dialysis lactate level (Table 2). There were no significant

differences in age, sex, baseline characteristics and comorbidities between the high

(pre-dialysis lactate $>4.2 \mathrm{mmol} / \mathrm{L}$ ) and low lactate (pre-dialysis lactate $\leqq 4.2 \mathrm{mmol} / \mathrm{L}$ )

groups. The 90-day mortality rate in the high lactate group was significantly higher

than that in the low lactate group. The prevalence rates of septic shock and SOFA

score on ICU admission in the high lactate group were significantly higher than those

in the low lactate group. Before dialysis, the high lactate group had significantly

higher $\mathrm{Hgb}$ and SOFA score but lower systolic blood pressure, daily UO and Glasgow

coma scale than the low lactate group.

\section{Analysis of Factors Associated with the Possibility of Dialysis Withdraw}

We also found that $12.4 \%$ of all of the patients and $26.3 \%$ of the survivors were

dialysis dependent at the end of the study. Figure 2 illustrates stratified cumulative

mortality rates according to pre-dialysis serum lactate level, and demonstrates that the

low lactate group had a significantly lower cumulative mortality rate than the high

lactate group. In Cox proportional hazards multivariate analysis taking mortality as a 
competing risk, pre-dialysis daily UO (subdistribution hazard ratio [sHR] 1.28, $\mathrm{p}=0.040), \mathrm{Hgb}(\mathrm{sHR} 1.09, \mathrm{p}=0.043), \mathrm{Na}(\mathrm{sHR} 1.02, \mathrm{p}=0.045)$, lactate $>4.2 \mathrm{mmol} / \mathrm{L}$ (sHR 0.07, p=0.009) and qSOFA score had independent prognostic significance for assessing the possibility of dialysis withdrawal. The sHRs for dialysis withdrawal calculated were severity dependent as per the stratification data of the pre-dialysis qSOFA scores and $0.14,0.15$, and 0.19 for qSOFA scores of 1,2 , and 3 , respectively. We further examined the interaction between lactate $>4.2$ and qSOFA score with regards to dialysis withdrawal. An attenuated trend of sHR was correlated with increasing qSOFA score in the patients with a serum lactate level $>4.2 \mathrm{mmol} / \mathrm{L}$ (Table 3). Figure 3 demonstrates the lower probability of dialysis withdrawal in the high lactate group compared to the low lactate group, taking mortality as a competing risk factor. Figure 4 shows plots of marginal effects for the probability of dialysis withdrawal (y axis) against predialysis qSOFA score ( $\mathrm{x}$ axis) according to a serum lactate level of $>4.2 \mathrm{mmol} / \mathrm{L}$ or $\leqq 4.2 \mathrm{mmol} / \mathrm{L}$. The interaction effects of lactate levels $\leqq 4.2 \mathrm{mmol} / \mathrm{L}$ and $>4.2 \mathrm{mmol} / \mathrm{L}$ with regards to the possibility of dialysis withdrawal and the severity of pre-dialysis qSOFA level are shown in Figure 4. The crossed lines on the graph support that there was an interaction effect, for which the significant 
interactions between dialysis withdrawal and pre-dialysis qSOFA score could be specified. The graph shows that the dialysis withdrawal rate was $44.8 \%$ higher in the low lactate group compared to the high lactate group when the pre-dialysis qSOFA score was $>1$.

\section{Sensitivity Analyses}

Table 4 shows the E-values for the point estimate and lower limit of the CI for pre-dialytic qSOFA score and lactate for mortality, separately. This analysis indicated no substantial unmeasured confounding (E-values for the point estimates [lower limits of the $\mathrm{CI}$ ] were 2.82 [1.00], 2.66 [1.00], 3.17 [1.00], and 2.32 [1.56] for qSOFA scores of 1, 2, 3, and lactate, respectively.

\section{Subgroup Analysis}

In subgroups analysis, the association between low lactate level and a lower risk of dialysis dependence remained consistent across diabetes, sepsis, fluid overload and uremia subgroups as the indication for initiating dialysis, while this association was more significant in the patients with hypertension and without coronary artery disease, congestive HF, history of PTCA or elective surgery (Figure 5).

\section{External Validation}


The results were then validated using the NEP-AKI-D database. A total of 240 type

1 CRS patients requiring dialysis were enrolled. In this multi-center database, the incidence rates of mortality, dialysis dependence and dialysis withdrawal at 90 days were $60.4 \%$ (145/240), $11.3 \%$ (27/240), and $28.3 \%$ (68/240), respectively. Multivariate analysis indicated that pre-dialysis serum lactate level was an independent predictor of 90-day mortality $(\mathrm{OR}, 2.276 \mathrm{p}=0.027)$ and dialysis withdrawal (sHR, $0.490 \mathrm{p}=0.047$ ) (Suppl. Tables).

\section{Discussion}

In this study, the 90-day mortality rate was $52.8 \%$ and the incidence rate of RRT dependence was $12.4 \%$ among type 1 CRS patents requiring dialysis for AKI. A lower pre-dialysis serum lactate level was associated with both a higher rate of survival and also a higher rate of dialysis withdrawal. Our results showed the considerable potential of serum lactate as a predictive biomarker for the probability of being successfully weaned from dialysis for AKI in type 1 CRS patents after considering death as a competing risk. We also showed that the interaction between qSOFA score and lactate level was associated with dialysis dependence in a disease 
severity-dependent manner. The results were consistent and further validated using

multi-center registration data (Suppl. Tables).

qSOFA score is a useful clinical tool for identifying infected patients likely to have life-threatening organ dysfunction. The third international consensus definitions for sepsis proposed that both qSOFA score and serum lactate levels are crucial clinical variables that can reflect the severity of acute illness in critically ill sepsis patients ${ }^{29}$. Recently, Wagner et al. reported a correlation between qSOFA score and the risk of worse outcomes in critically ill patients with acute decompensated $\mathrm{HF}^{30}$. We found that in addition to septic disease, the interaction between qSOFA and lactate level could predict the outcomes in type $1 \mathrm{CRS}$ patients. In our model, less unmeasured confounding is needed to explain away the observed association (Table 4).

To estimate the occurrence of dialysis dependence, we used a competing risk model, which could more accurately estimate the risk of dialysis dependence while considering that a high percentage of patients may die before the end of the 90-day study follow-up period ${ }^{31}$. The early recognition of patients at risk of RRT dependence and mortality may allow for timely and targeted interventions for these patients. Our 
results identified that daily $\mathrm{UO}, \mathrm{Hgb}, \mathrm{Na}$, lactate $>4.2 \mathrm{mmol} / \mathrm{L}$ and qSOFA score determined before dialysis were independent predictors for the possibility of dialysis withdrawal (Table 3).

Lactic acid is produced in hypoxic conditions and high glycolytic activity ${ }^{32,33}$. Multiple inflammatory mechanisms are involved in the accumulation of lactate acid, including direct cellular injury, inflammation-induced injury, microcirculatory dysfunction, arterial hypotension and reduced oxygen extraction from surrounding tissues $^{11,34}$. In type $1 \mathrm{CRS}$, the principal causes of kidney abnormalities are low cardiac output, decreased renal blood flow and secondary tubular hypoxic injury ${ }^{35}$. The level of serum lactate may reflect both the severity of disease and also the degree of anaerobic metabolism, further leading to different renal outcomes in this clinical setting (Figure 4$)^{34}$.

The Third International Consensus Definitions for Sepsis and Septic Shock had proposed that elevated serum lactate levels represent an important marker of "cryptic shock" in the absence of hypotension, and the clinical diagnosis of septic shock should be based on the combination of hyperlactatemia with fluid-resistant hypotension ${ }^{29}$. Recently, the association between the elevation of serum lactate levels 
and ventricular dysfunction in septic patients was reported ${ }^{36}$. Nevertheless, to the best of our knowledge, there is still lacking of information about the prognostic value for hyperlactatemia in type 1 CRS patient. In the current study, the patients with a high and low pre-dialysis serum lactate level had significantly different survival rates and cumulative kidney withdrawal rates (Table 2 and Figure 3). Moreover, we found that the associations between hyperlactatemia and dialysis dependence were consistent in the patients with and without sepsis (Figure 5).

In the literature, there is currently no general consensus on the correlation between exact lactate levels and poor prognosis ${ }^{37}$. An increase in serum lactate $>2$ $\mathrm{mmol} / \mathrm{L}$ had been reported to play a key role in the diagnosis of cardiogenic shock and septic shock ${ }^{37}$. We further found that a serum lactate level $>4.2 \mathrm{mmol} / \mathrm{L}$ could reflect the dynamic aspects of disease severity and provide superior information on the prognosis of our patients with type 1 CRS (Figure 1). The cut-off serum lactate value in our study is similar to the component of IABP-SHOCK II risk score of 5 $\mathrm{mmol} / \mathrm{L}$ for severe cardiogenic shock ${ }^{38}$. Cardiac dysfunction results in systemic congestion, which in turn causes absolute or relative arterial hypovolemia and upregulates the sympathetic system and the release of arginine vasopressin, thereby 
exacerbating positive fluid balance and further impairing cardiac function because of a progressive increase in cardiac filling pressure. Type 1 CRS patients are susceptible to a vicious circle of reduced cardiac performance and fluid overload ${ }^{35,39}$. Lactate is a unique HF marker because it reflects the dynamic metabolic status of the patient rather than the static degree of myocardial damage or dysfunction (such as NTproBNP or troponin I) ${ }^{40}$. Interestingly, the subgroup analysis in our study showed that the association between low lactate level and a lower risk of dialysis was more prominent in the patients without coronary artery disease, congestive HF, history of PTCA or elective surgery. This finding suggests that serum lactate level may be more predictive in patients without a history of prior cardiovascular diseases (Figure 5).

In spite of the encouraging results observed in this study, several potential limitations should also be recognized. First, the fact that our study involved patients of the same ethnicity limits the generalizability of the findings to other hospitals with different patient populations. Second, the predictive value of lactate clearance in the risk of mortality has been well documented in many clinical scenarios ${ }^{12,33}$. However, serum lactate level was not measured sequentially in this study, and the role of sequential measurements of serum lactate and lactate clearance may be more useful 
prognostic markers than initial lactate alone. We also acknowledge that the observational nature of the study without a pre-specified protocol for the intervention cannot conclude causal relationships. Therefore, we can only speculate that serum lactate level may be a decision-making variable, and further studies are needed to validate our results. We recorded the worst SOFA score within 24 hours before the initiation of dialysis. Although this approach is consistent with clinical practice, the daily SOFA or qSOFA score may not reflect the value immediately before the initiation of RRT.

In conclusion, more than half of the type 1 CRS patients in this study died and $34.8 \%$ were successfully weaned from dialysis for AKI. Our results showed that a serum lactate level $>4.2 \mathrm{mmol} / \mathrm{L}$ before dialysis should be considered to be an independent risk factor for 90-day mortality and dialysis dependence, regardless of the presence or absence of sepsis. We confirmed that serum lactate level is accurate and capable of forecasting the prognosis along with qSOFA severity for clinical decision-making for treating type 1 CRS patients.

\section{Patients and Methods}




\section{Ethics statement and Registration of the Clinical Trial}

This study was conducted in full compliance with the ethical principles of the Declaration of Helsinki and was consistent with Good Clinical Practice guidelines and the applicable local regulatory requirements. The trial was registered prior to patient enrollment at clinicaltrials.gov (NCT01503710, Principal investigator: V C Wu, Date of registration: 02/28/2012). The study was approved by the Institutional Review Board of National Taiwan University Hospital (201407076RINA). Patients who met the inclusion criteria were invited to participate in this study on their first day of admission to the intensive care unit (ICU). Trained physicians evaluated the patients' mental status during the screening and proceeded to perform informed consent procedures. A written informed consent was obtained from all mentally competent subjects prior to their participation.

\section{Study Design}

We prospectively enrolled patients with chronic kidney disease undergoing RRT for AKI in multiple intensive care units (ICUs) from the National Taiwan University Study Group on Acute Renal Failure ${ }^{13-17}$. According to the consensus definition of the Acute Dialysis Quality Initiative (ADQI) group, type 1 CRS was defined as an abrupt 
worsening of cardiac function leading to acute impairment of kidney function ${ }^{2}$. From August 2011 to January 2019, type 1 CRS patients who required RRT after ICU admission were prospectively enrolled. The exclusion criteria were: age $<18$ years, previous nephrectomy, renal transplantation or prior RRT treatment, ICU or hospital length of stay of respectively $<2$ days and $>180$ days during the index hospitalization, and AKI caused by kidney surgically induced injury, vasculitis, obstruction, glomerulonephritis, interstitial nephritis, hemolytic uremic syndrome, or thrombotic thrombocytopenic purpura.

\section{Patient Enrollment and Data Collection}

The cardiac reasons for ICU admission included: acute coronary syndrome, acute decompensated HF, or impending HF with the need for intensive monitoring. All data were collected prospectively, including data on the patients' sex, age, comorbidities, the primary reason for ICU admission, underlying heart disease, disease severity and clinical parameters before dialysis.

Baseline serum creatinine ( $\mathrm{sCr}$ ) was defined as the nadir value obtained after the previous admission in those who had more than one admission within 1 year before the index admission, or the mean outpatient value over the 180 days before the index 
admission in those with no previous admission ${ }^{18,19}$. The RRT modality in each patient was initially chosen by the attending physician and adapted according to hemodynamic evaluation and evolution by a critical care nephrologist.

\section{Indications for Dialysis}

Indications for initiating RRT included one or more of the following: (1) azotemia (i.e. blood urea nitrogen $>80 \mathrm{mg} / \mathrm{dL}$ and $\mathrm{sCr}>2 \mathrm{mg} / \mathrm{dL}$ ) with uremic symptoms (encephalopathy, pericarditis, or pleuritis); (2) oliguria (urine output $<400 \mathrm{~mL} / 24 \mathrm{~h}$ ) or anuria refractory to diuretics; (3) fluid overload refractory to diuretics with a central venous pressure $>12 \mathrm{mmHg}$ or pulmonary edema with $\mathrm{PaO}_{2} / \mathrm{FiO}_{2}<300$ $\mathrm{mmHg}$; (4) hyperkalemia (serum potassium level $>5.5 \mathrm{mmol} / \mathrm{L}$ ) refractory to medical treatment; and (5) metabolic acidosis $(\mathrm{pH}<7.2 \text { in arterial blood })^{13,20,21}$.

\section{Outcome Assessments}

The primary clinical endpoints were 90-day mortality after hospital discharge and withdrawal from RRT in the survivors. All patients were followed until death or for 90 days after discharge, whichever occurred first. Successful withdrawal from dialysis was defined as surviving without dialysis at the end of study. Patients who died within 14 days after being weaned from dialysis were classified into the mortality group 
rather than the recovery group, because biological kidney recovery without survival is not patient-centered ${ }^{22}$. Patients who received acute dialysis and planned to receive palliative care were excluded.

\section{External Validation}

We used the database of the Nationwide Epidemiology and Prognosis of Dialysisrequiring Acute Kidney Injury (NEP-AKI-D) study for further external validation ${ }^{23}$. The NEP-AKI-D study was designed to enroll critically ill adult patients with AKI-D receiving RRT in the ICUs of both tertiary medical centers and regional hospitals located in the four geographical regions (north, middle, south, and east) of Taiwan. The four months during which the participants were enrolled were October 2014, and January, April, and July 2015, reflecting the seasons in Taiwan. After the enrollment process, we followed the clinical courses and documented the outcomes of these enrolled participants until death or 90 days following hospital discharge. The study was approved by the National Research Program for Biopharmaceuticals (NRPB)Institutional Review Board (IRB) (NRPB2014050014) and the IRBs of all participating hospitals ${ }^{24}$.

\section{Statistical Analysis}


Continuous data were expressed as mean \pm standard deviation. Categorical data were expressed as number (percentage), and the $\chi^{2}$ or Fisher's exact test was used for comparisons. All variables were tested for normal distribution using the KolmogorovSmirnov test. The Student's t-test was used to compare the means of continuous variables and normally distributed data; otherwise, the Mann-Whitney U test was used. The $\chi^{2}$ test was used to compare categorical data. A generalized additive model was plotted and adjusted for comorbidities, sex and age in individual patients ${ }^{25,26}$. The model incorporated subject-specific random effects, expressed as the logarithm of the odd (logit), and the optimal cut-off value was defined as a log odds value of zero ${ }^{27}$. The variables were assessed in multivariate analysis using a Fine-Gray subdistribution hazard model to estimate subdistribution hazard ratios (sHRs) for the possibility of dialysis withdrawal, taking mortality as a competing risk. The significance levels for entry (SLE) and stay (SLS) were conservatively set to 0.15 . We computed E-values using the methodology proposed by VanderWeele and Ding ${ }^{28}$. Specifically, the Evalues quantify what the risk ratio would need to be for unmeasured confounders to explain away the observed associations of pre-dialytic qSOFA score and lactate for mortality in the present study. All statistical tests were two-tailed, and a $p$ value of $<$ 
0.05 was considered to be statistically significant. All analyses were performed using

SPSS software (version 20, IBM, Armonk, NY), R software (version 3.4.2, Free

Software Foundation, Inc., Boston, MA), and MedCalc Statistical Software (version

15.11.3, MedCalc Software bvba, Oostende, Belgium; https://www.medcalc.org; 2015).

\section{Data Availability Statement}

The datasets used and/or analyzed during the current study are available from the corresponding author on reasonable request.

\section{References}

1 Initiative, A. D. Q. consensus group. Cardio-renal syndromes: report from the consensus conference of the Acute Dialysis Quality Initiative. Europ Heart J 31, 703-711 (2010).

2 Ronco, C., Cicoira, M. \& McCullough, P. A. Cardiorenal syndrome type 1: pathophysiological crosstalk leading to combined heart and kidney dysfunction in the setting of acutely decompensated heart failure. Journal of the American College of Cardiology 60, 1031-1042 (2012).

3 Li, J. et al. Is the mean platelet volume a predictive marker of a high inhospital mortality of acute cardiorenal syndrome patients receiving continuous renal replacement therapy? Medicine 97 (2018).

4 Shirakabe, A. et al. Long-term prognostic impact after acute kidney injury in patients with acute heart failure. International heart journal 53, 313-319 
(2012).

5 Wellen, K. E. \& Hotamisligil, G. S. Inflammation, stress, and diabetes. The Journal of clinical investigation 115, 1111-1119 (2005).

6 Dries, D. L., Exner, D. V., Domanski, M. J., Greenberg, B. \& Stevenson, L. W. The prognostic implications of renal insufficiency in asymptomatic and symptomatic patients with left ventricular systolic dysfunction. Journal of the American College of Cardiology 35, 681-689 (2000).

$7 \quad \mathrm{Wu}, \mathrm{B}$. et al. Initiation and cessation timing of renal replacement therapy in patients with type 1 cardiorenal syndrome: An observational study.

Cardiorenal medicine 7, 118-127 (2017).

8 Mitaka, C. et al. Polymyxin B hemoperfusion prevents acute kidney injury in sepsis model. journal of surgical research 201, 59-68 (2016).

9 Metra, M. et al. The role of plasma biomarkers in acute heart failure. Serial changes and independent prognostic value of NT-proBNP and cardiac troponin-T. European journal of heart failure 9, 776-786 (2007).

10 Wittayachamnankul, B., Chentanakij, B., Sruamsiri, K. \& Chattipakorn, N. The role of central venous oxygen saturation, blood lactate, and central venous-to-arterial carbon dioxide partial pressure difference as a goal and prognosis of sepsis treatment. Journal of critical care 36, 223-229 (2016).

11 Albright, C. M., Ali, T. N., Lopes, V., Rouse, D. J. \& Anderson, B. L. Lactic acid measurement to identify risk of morbidity from sepsis in pregnancy. American Journal of Perinatology 32, 481-486 (2015).

12 da Hora Passos, R. et al. Lactate clearance is associated with mortality in septic patients with acute kidney injury requiring continuous renal replacement therapy: a cohort study. Medicine 95 (2016).

$13 \mathrm{Wu}, \mathrm{V}$. C. et al. Early renal replacement therapy in patients with postoperative acute liver failure associated with acute renal failure: effect on postoperative outcomes. Journal of the American College of Surgeons 205, 266-276 (2007).

$14 \mathrm{Wu}, \mathrm{V}$. C. et al. Risk factors of early redialysis after weaning from postoperative acute renal replacement therapy. Intensive care medicine $\mathbf{3 4}$, 101-108 (2008).

15 Shiao, C. C. et al. Late initiation of renal replacement therapy is associated with worse outcomes in acute kidney injury after major abdominal surgery. Crit Care 13, R171 (2009).

$16 \mathrm{Wu}, \mathrm{V}$. C. et al. Sustained low-efficiency dialysis versus continuous veno- 
venous hemofiltration for postsurgical acute renal failure. Am J Surg 199, 466$476(2010)$.

17 Huang, T. M. et al. Preoperative proteinuria predicts adverse renal outcomes after coronary artery bypass grafting. J Am Soc Nephrol 22, 156-163 (2011).

$18 \mathrm{Wu}, \mathrm{V}$. C. et al. Acute-on-chronic kidney injury at hospital discharge is associated with long-term dialysis and mortality. Kidney Int 80, 1222-1230 (2011).

19 Shu, K. H. et al. Urinary pi-glutathione S-transferase Predicts Advanced Acute Kidney Injury Following Cardiovascular Surgery. Sci Rep 6, 26335 (2016).

20 Lin, Y. F. et al. A modified sequential organ failure assessment score to predict hospital mortality of postoperative acute renal failure patients requiring renal replacement therapy. Blood Purif 26, 547-554 (2008).

21 Shiao, C. C. et al. U-curve association between timing of renal replacement therapy initiation and in-hospital mortality in postoperative acute kidney injury. PloS one 7, e42952 (2012).

22 Kellum, J. A. How can we define recovery after acute kidney injury? Considerations from epidemiology and clinical trial design. Nephron Clin Pract 127, 81-88 (2014).

23 Shiao, C. C. et al. Nationwide epidemiology and prognosis of dialysisrequiring acute kidney injury (NEP-AKI-D) study: Design and methods. Nephrology 21, 758-764 (2016).

24 Shiao, C.-C. et al. Association between regional economic status and renal recovery of dialysis-requiring acute kidney injury among critically ill patients. Sci. Rep. 10, 1-10 (2020).

$25 \mathrm{Wu}, \mathrm{V}$. C. et al. Endothelial progenitor cells in primary aldosteronism: a biomarker of severity for aldosterone vasculopathy and prognosis. $J$ Clin Endocrinol Metab 96, 3175-3183 (2011).

$26 \mathrm{Wu}, \mathrm{V}$. C. et al. Effect of diuretic use on 30-day postdialysis mortality in critically ill patients receiving acute dialysis. PloS one 7, e30836 (2012).

27 Hin, L. Y., Lau, T. K., Rogers, M. S. \& Chang, A. M. Dichotomization of continuous measurements using generalized additive modelling--application in predicting intrapartum caesarean delivery. Stat Med 18, 1101-1110 (1999).

28 VanderWeele, T. J. \& Ding, P. Sensitivity analysis in observational research: introducing the E-value. Ann. Intern. Med. 167, 268-274 (2017).

29 Singer, M. et al. The third international consensus definitions for sepsis and 
septic shock (Sepsis-3). Jama 315, 801-810 (2016).

30 Wagner, T. et al. qSOFA Score Is Useful to Assess Disease Severity in Patients With Heart Failure in the Setting of a Heart Failure Unit (HFU). Frontiers in cardiovascular medicine 7 (2020).

31 Noordzij, M. et al. When do we need competing risks methods for survival analysis in nephrology? Nephrology Dialysis Transplantation 28, 2670-2677 (2013).

32 Pucino, V., Cucchi, D. \& Mauro, C. Lactate transporters as therapeutic targets in cancer and inflammatory diseases. Expert opinion on therapeutic targets 22, 735-743 (2018).

33 Ryoo, S. M. et al. Lactate level versus lactate clearance for predicting mortality in patients with septic shock defined by sepsis-3. Critical care medicine 46, e489-e495 (2018).

34 Prowle, J. R., Kirwan, C. J. \& Bellomo, R. Fluid management for the prevention and attenuation of acute kidney injury. Nature Reviews Nephrology 10, 37 (2014).

35 Valika, A. A. \& Costanzo, M. R. The acute cardiorenal syndrome type I: considerations on physiology, epidemiology, and therapy. Current heart failure reports 11, 382-392 (2014).

36 Innocenti, F. et al. Prognostic stratification in septic patients with overt and cryptic shock by speckle tracking echocardiography. Internal and Emergency Medicine, 1-8 (2020).

37 Van Diepen, S. et al. Contemporary management of cardiogenic shock: a scientific statement from the American Heart Association. Circulation 136, e232-e268 (2017).

38 Thiele, H. et al. Intraaortic balloon pump in cardiogenic shock complicating acute myocardial infarction: long-term 6-year outcome of the randomized IABP-SHOCK II trial. Circulation 139, 395-403 (2019).

39 McCullough, P. A. et al. in ADQI Consensus on AKI Biomarkers and Cardiorenal Syndromes Vol. 182 82-98 (Karger Publishers, 2013).

40 Biegus, J. et al. Persistent hyperlactataemia is related to high rates of inhospital adverse events and poor outcome in acute heart failure. Kardiologia polska 77, 355-362 (2019). 


\section{Acknowledgements}

This study was supported by grants from the Taiwan National Science Council [104-

2314-B-002-125-MY3, 106-2314-B-002 -166 -MY3,107-2314-B-002-026-MY3],

National Health Research Institutes [PH-102-SP-09], National Taiwan University

Hospital [106-FTN20, 106-P02, UN106-014, 106-S3582, 107-S3809, 107-

T02,PC1246,VN109-09,109-S4634,UN109-041] and Ministry of Science and

Technology (MOST) of the Republic of China (Taiwan) [grant number, MOST 106-

2321-B-182-002, MOST 107-2321-B-182-004, MOST 108-2321-B-182-003, MOST

109-2321-B-182-001]. The authors also thank all participants of NSARF and CAKs

as well as the staff of the National Health Research Institute and Harvard Statistics.

(The details of the members of CAKs can be downloaded here:

http://links.lww.com/MD/B298).

\section{Authors' contributions}

VCW contributed to the conception, design and interpretation of data. HCP contributed

to collecting data and manuscript drafting. VCW provided patient information and participated in the design and coordination and helped to draft the manuscript. VCW 
and HCP contributed to provide intellectual content of the work and were involved in editing and revising the manuscript. All authors discussed, contributed to, and approved the final manuscript version.

\section{Additional Information}

Competing interests: The authors declare no competing financial interests.

Figure legends 
Figure 1. Generalized additive model plot for the probability of 90-day mortality

against serum lactate levels at the initiation of dialysis. The generalized additive model plot was incorporated with subject-specific random effects expressed as the logarithm of the odds (logit). The probability of outcome events was constructed with lactate levels averaging zero over the range of the data, i.e. lactate $=4.24 \mathrm{ng} / \mathrm{mL}$.

Abbreviations: AKI, acute kidney injury; ICU, intensive care unit; qSOFA, quick Sequential Organ Failure Assessment

II Pre-dialysis lactate level $\leqq 4.2 \mathrm{mmol}$ was defined as low lactate Pre-dialysis lactate level $>4.2 \mathrm{mmol}$ was defined as high lactate

Figure 2. Cox proportional hazard plots stratified by pre-dialysis serum lactate level for assessing the probability of mortality. All relevant covariates, including characteristics, comorbidities and laboratory data at ICU admission, etiology of AKI, indication for dialysis, dialysis modality, qSOFA score, and plasma lactate level at dialysis, and some of their interactions including those listed in Table 1 were put on a selected variable list to predict the outcome of interest. 
Figure 3. Cox proportional hazard plots stratified by pre-dialysis serum lactate level for assessing probability of dialysis withdraw, taking mortality as a competing risk ${ }^{\mathrm{II}}$.

${ }^{\mathbb{I}}$ Pre-dialysis lactate level $\leqq 4.2 \mathrm{mmol}$ was defined as low lactate Pre-dialysis lactate level > $4.2 \mathrm{mmol}$ was defined as high lactate

Figure 4. Marginal effects of the interaction between probability of dialysis withdrawal and predialysis qSOFA score according to a high (> $4.2 \mathrm{mmol} / \mathrm{L})$ or low ( $\leqq 4.2 \mathrm{mmol} / \mathrm{L})$ predialysis serum lactate level. The crossed blue and red lines supported that there was an interaction effect, in which an impressive interaction between the probability of dialysis withdrawal and predialysis qSOFA score was confirmed under the influence of a high and low lactate level. The graph showed that the probability of dialysis withdrawal was significantly higher for the acute dialysis patients with a pre-dialysis serum lactate level $\leqq 4.2 \mathrm{mmol} / \mathrm{L}(\mathrm{p}<0.001)$. 
Figure 5. Forest plot depicting subgroup analysis of dialysis withdrawal

compared with high and low pre-dialysis serum levels of lactate, taking mortality

as a competing risk ${ }^{\mathrm{II}}$.

Abbreviations: CAD, coronary artery disease; PTCA, percutaneous transluminal coronary angioplasty;

II Pre-dialysis lactate level $\leqq 4.2 \mathrm{mmol}$ was defined as low lactate

Pre-dialysis lactate level $>4.2 \mathrm{mmol}$ was defined as high lactate

Table 1 Baseline Characteristics of enrollee 


\begin{tabular}{|c|c|c|c|c|c|}
\hline & $\begin{array}{l}\text { Total patient } \\
\qquad(\mathrm{n}=500)\end{array}$ & $\begin{array}{l}\text { Mortality } \\
(n=264)\end{array}$ & $\begin{array}{c}\text { dialysis } \\
\text { dependence } \\
\quad(n=62)\end{array}$ & $\begin{array}{l}\text { dialysis } \\
\text { withdraw } \\
(n=174)\end{array}$ & ANOVA \\
\hline \multicolumn{6}{|l|}{ Demographic factors } \\
\hline Age, years & $61.4 \pm 16.3$ & $62.8 \pm 16.2$ & $63.4 \pm 15.1$ & $58.4 \pm 16.4$ & 0.011 \\
\hline Male gender & $360(72.0 \%)$ & $191(72.4 \%)$ & $41(66.1 \%)$ & $128(73.6 \%)$ & 0.526 \\
\hline Baseline SCr & $2.0 \pm 1.7$ & $1.8 \pm 1.4$ & $2.9 \pm 2.3$ & $1.9 \pm 1.7$ & $<.0001$ \\
\hline Smoking & $1(20.8 \%)$ & $54(20.5 \%)$ & $12(19.4 \%)$ & $38(21.8 \%)$ & 0.900 \\
\hline Hypertension & $283(56.6 \%)$ & $145(54.9 \%)$ & $39(62.9 \%)$ & $99(56.9 \%)$ & 0.519 \\
\hline Diabetes mellitus & $215(43.0 \%)$ & $101(38.3 \%)$ & $37(59.7 \%)$ & $77(44.3 \%)$ & 0.008 \\
\hline Coronary artery disease & $196(39.2 \%)$ & $102(38.6 \%)$ & $28(45.2 \%)$ & $66(37.9 \%)$ & 0.584 \\
\hline Congestive heart failure & $370(74.0 \%)$ & $191(72.4 \%)$ & $47(75.8 \%)$ & $132(75.9 \%)$ & 0.673 \\
\hline NYHA FC 1 & $140(28.0 \%)$ & $75(28.4 \%)$ & $14(22.6 \%)$ & $51(29.3 \%)$ & 0.585 \\
\hline NYHA FC 2 & $87(17.4 \%)$ & $44(16.7 \%)$ & $11(17.7 \%)$ & $32(18.4 \%)$ & 0.895 \\
\hline NYHA FC 3 & $77(15.4 \%)$ & $42(15.9 \%)$ & $8(12.9 \%)$ & $27(15.5 \%)$ & 0.839 \\
\hline NYHA FC 4 & $66(13.2 \%)$ & $30(11.4 \%)$ & $14(22.6 \%)$ & $22(12.7 \%)$ & 0.061 \\
\hline Chalson score & $5.3 \pm 2.5$ & $4.5 \pm 2.6$ & $4.7 \pm 2.3$ & $4.0 \pm 2.5$ & 0.066 \\
\hline History of PTCA & $137(27.4 \%)$ & $70(26.5 \%)$ & $21(33.9 \%)$ & $46(26.4 \%)$ & 0.475 \\
\hline Single vessel & $13(2.6 \%)$ & $7(2.7 \%)$ & $1(1.6 \%)$ & $5(2.9 \%)$ & 1.000 \\
\hline Two vessels & $29(5.8 \%)$ & $10(3.8 \%)$ & $6(9.7 \%)$ & $13(7.5 \%)$ & 0.103 \\
\hline Three vessels & $95(19.0 \%)$ & $53(20.1 \%)$ & $14(22.6 \%)$ & $28(16.1 \%)$ & 0.434 \\
\hline With stents & $22(4.4 \%)$ & $10(3.8)$ & $9(14.5)$ & $13(7.5)$ & 0.006 \\
\hline History of CABG & $70(14.0 \%)$ & $41(15.5 \%)$ & $5(8.1 \%)$ & $24(13.8 \%)$ & 0.311 \\
\hline Single vessel & $5(1.0 \%)$ & $3(1.1 \%)$ & $0(0.0 \%)$ & $2(1.2 \%)$ & 1.000 \\
\hline Two vessels & $13(2.6 \%)$ & $6(2.3 \%)$ & $2(3.2 \%)$ & $5(2.9 \%)$ & 0.784 \\
\hline Three vessels & $52(10.4 \%)$ & $32(12.1 \%)$ & $3(4.8 \%)$ & $17(9.8 \%)$ & 0.226 \\
\hline ICU Admission SOFA & $10.51 \pm 4.02$ & $11.26 \pm 4.04$ & $9.38 \pm 3.77$ & $9.76 \pm 3.85$ & $<.0001$ \\
\hline ICU Admission qSOFA & $1.3 \pm 0.8$ & $1.5 \pm 0.8$ & $1.1 \pm 0.8$ & $1.2 \pm 0.8$ & $<.0001$ \\
\hline
\end{tabular}

\section{Etiology of AKI}

\begin{tabular}{llllll}
\hline Cardiogenic shock & $170(34.0 \%)$ & $87(33.0 \%)$ & $24(38.7 \%)$ & $59(33.9 \%)$ & 0.690 \\
\hline Sepsis & $263(52.6 \%)$ & $154(58.3 \%)$ & $24(38.7 \%)$ & $85(48.9 \%)$ & 0.010 \\
\hline Septic shock & $145(29.0 \%)$ & $86(32.5 \%)$ & $11(17.74 \%)$ & $48(27.6 \%)$ & 0.060 \\
\hline
\end{tabular}

\section{Clinical parameters before dialysis}

$\begin{array}{llllll}\mathrm{SBP}, \mathrm{mmHg} & 108.0 \pm 26.8 & 102.1 \pm 24.6 & 120.0 \pm 30.3 & 112.5 \pm 26.6 & <.0001\end{array}$




\begin{tabular}{lccccc}
\hline Daily UO $(\mathrm{log}), \mathrm{ml}$ & $2.2 \pm 0.9$ & $2.0 \pm 1.0$ & $2.3 \pm 0.9$ & $2.4 \pm 0.8$ & $<.0001$ \\
\hline $\mathrm{GCS}$ & $9.6 \pm 4.5$ & $8.2 \pm 4.5$ & $11.6 \pm 3.6$ & $11.0 \pm 4.3$ & $<.0001$ \\
\hline $\mathrm{Hgb}$ & $10.68 \pm 2.31$ & $10.73 \pm 2.34$ & $9.63 \pm 1.77$ & $10.99 \pm 2.34$ & $<.0001$ \\
\hline $\mathrm{Na}, \mathrm{mmol} / \mathrm{L}$ & $139.3 \pm 7.7$ & $139.4 \pm 8.0$ & $137.3 \pm 6.2$ & $139.8 \pm 7.7$ & 0.089 \\
\hline $\mathrm{K}, \mathrm{mEq} / \mathrm{L}$ & $4.4 \pm 0.9$ & $4.3 \pm 0.9$ & $4.3 \pm 0.6$ & $4.4 \pm 0.9$ & 0.337 \\
\hline Lactate, $\mathrm{mmol} / \mathrm{L}$ & $5.7 \pm 4.5$ & $6.9 \pm 4.8$ & $3.7 \pm 2.8$ & $4.7 \pm 3.9$ & $<.0001$ \\
\hline SOFA & $12.11 \pm 3.77$ & $13.31 \pm 3.50$ & $9.95 \pm 3.58$ & $11.05 \pm 3.59$ & $<.0001$ \\
\hline qSOFA & $2.0 \pm 0.6$ & $2.1 \pm 0.6$ & $1.9 \pm 0.7$ & $1.8 \pm 0.7$ & $<.0001$ \\
\hline
\end{tabular}

Abbreviations: AKI, acute kidney injury; Hgb, hemoglobin; CABG, coronary artery bypass graft; FC, functional class; GCS, Glasgow coma scale; ICU, intensive care unit; MI, myocardial infarction; NYHA, New York Heart Association; PTCA, percutaneous transluminal coronary angioplasty; qSOFA, quick Sequential Organ Failure Assessment; SBP, systolic blood pressure; SCr, serum creatinine; SOFA, Sequential Organ Failure Assessment; TPN, total parental nutrition; UO, urine output 
Table 2 Basic characteristics of enrollee divided by pre-dialysis lactate level

\begin{tabular}{|c|c|c|c|}
\hline Predictors & $\begin{array}{l}\text { Pre-dialysis } \\
\text { lactate }>4.2 \mathrm{mmol} / \mathrm{L}^{\#} \\
(\mathrm{n}=288)\end{array}$ & $\begin{array}{l}\text { Pre-dialysis_ } \\
\text { lactate } \leqq 4.2 \mathrm{mmol} / \mathrm{L}^{\#} \\
(\mathrm{n}=212)\end{array}$ & P-value \\
\hline \multicolumn{4}{|l|}{ Demographic factors } \\
\hline Age, years & $60.8 \pm 16.9$ & $62.0 \pm 15.4$ & 0.418 \\
\hline Male gender & $205(71.2 \%)$ & $155(73.1 \%)$ & 0.634 \\
\hline Baseline SCr, (mg/dL) & $1.9 \pm 1.7$ & $2.0 \pm 1.6$ & 0.468 \\
\hline Smoking & $57(19.8 \%)$ & $47(22.2 \%)$ & 0.517 \\
\hline Diabetes mellitus & $115(39.9 \%)$ & $100(47.2 \%)$ & 0.106 \\
\hline Hypertension & $155(53.8 \%)$ & $128(60.4 \%)$ & 0.144 \\
\hline Coronary artery disease & $105(36.5 \%)$ & $91(42.9 \%)$ & 0.143 \\
\hline Congestive heart failure & $214(74.3 \%)$ & $156(73.6 \%)$ & 0.856 \\
\hline NYHA FC 1 & $85(29.5 \%)$ & $55(25.9 \%)$ & 0.380 \\
\hline NYHA FC 2 & $50(17.4 \%)$ & $37(17.5 \%)$ & 0.979 \\
\hline NYHA FC 3 & $43(14.9 \%)$ & $34(16.0 \%)$ & 0.735 \\
\hline NYHA FC 4 & $36(12.5 \%)$ & $30(14.2 \%)$ & 0.590 \\
\hline Chalson score & $4.0 \pm 2.5$ & $4.5 \pm 2.6$ & 0.668 \\
\hline History of PTCA & $74(25.7 \%)$ & $63(29.7 \%)$ & 0.319 \\
\hline Single vessel & $5(1.7 \%)$ & $8(3.8 \%)$ & 0.157 \\
\hline Two vessels & $14(4.9 \%)$ & $15(7.1 \%)$ & 0.295 \\
\hline Three vessels & $55(19.1 \%)$ & $40(18.9) \%$ & 0.949 \\
\hline With stents & $15(5.2 \%)$ & $17(8.0 \%)$ & 0.205 \\
\hline History of CABG & $40(13.9 \%)$ & $30(14.2 \%)$ & 0.934 \\
\hline Single vessel & $1(0.4 \%)$ & $4(1.9 \%)$ & 0.168 \\
\hline Two vessels & $7(2.4 \%)$ & $6(2.8 \%)$ & 0.781 \\
\hline Three vessels & $32(11.1 \%)$ & $20(9.4 \%)$ & 0.544 \\
\hline ICU Admission SOFA & $10.94 \pm 4.20$ & $9.91 \pm 3.69$ & 0.004 \\
\hline ICU Admission qSOFA & $1.3 \pm 0.9$ & $1.3 \pm 0.8$ & 0.770 \\
\hline \multicolumn{4}{|l|}{ Etiology of AKI } \\
\hline Cardiogenic shock & $88(30.6 \%)$ & $82(38.7 \%)$ & 0.058 \\
\hline Sepsis & $157(54.5 \%)$ & $106(50.0 \%)$ & 0.318 \\
\hline Septic shock & $111(38.5 \%)$ & $34(16.0 \%)$ & $<.0001$ \\
\hline
\end{tabular}


Clinical parameters before dialysis

\begin{tabular}{llll}
\hline $\mathrm{SBP}, \mathrm{mmHg}$ & $103.5 \pm 26.4$ & $114.0 \pm 26.2$ & $<.0001$ \\
\hline Daily UO $(\mathrm{log}), \mathrm{ml}$ & $2.1 \pm 1.0$ & $2.4 \pm 0.9$ & 0.001 \\
\hline $\mathrm{GCS}$ & $8.8 \pm 4.8$ & $10.8 \pm 4.0$ & $<.0001$ \\
\hline $\mathrm{Hgb}$ & $11(2.5 \%)$ & $10(1.9 \%)$ & 0.002 \\
\hline $\mathrm{Na}, \mathrm{mmol} / \mathrm{L}$ & $139.6 \pm 7.0$ & $138.9 \pm 8.6$ & 0.349 \\
\hline $\mathrm{K}, \mathrm{mEq} / \mathrm{L}$ & $4.3 \pm 0.9$ & $4.4 \pm 0.8$ & 0.052 \\
\hline SOFA & $12.8 \pm 3.8$ & $11.2 \pm 3.5$ & $<.0001$ \\
\hline qSOFA & $2.0 \pm 0.6$ & $2.0 \pm 0.6$ & 0.932 \\
\hline 90-day outcome & & & \\
\hline Mortality & $179(62.2 \%)$ & $85(40.1 \%)$ & $<.0001$ \\
\hline RRT dependence & $23(8.0 \%)$ & $30(14.2 \%)$ & 0.063 \\
\hline
\end{tabular}

Abbreviations: AKI, acute kidney injury; Hgb, hemoglobin; CABG, coronary artery bypass graft; FC, functional class; GCS, Glasgow coma scale; ICU, intensive care unit; MI, myocardial infarction; NYHA, New York Heart Association; PTCA, percutaneous transluminal coronary angioplasty; qSOFA, quick Sequential Organ Failure Assessment; SBP, systolic blood pressure; SCr, serum creatinine; SOFA, Sequential Organ Failure Assessment; TPN, total parental nutrition; UO, urine output 
Table 3 Cox proportional hazards models depicting the possibility of dialysis withdraw, taking mortality as a competing risk.

\begin{tabular}{|c|c|c|}
\hline Predictors & sHR with $95 \% \mathrm{CI}$ & $\mathrm{p}$-value \\
\hline \multicolumn{3}{|l|}{ Demographic factors } \\
\hline Age & $0.99(0.98-1.00)$ & 0.084 \\
\hline Male gender & $1.03(0.70-1.52)$ & 0.885 \\
\hline Baseline SCr & $0.95(0.87-1.05)$ & 0.351 \\
\hline Smoking & $0.97(0.62-1.51)$ & 0.889 \\
\hline Hypertension & $0.99(0.69-1.42)$ & 0.961 \\
\hline Diabetes mellitus & $1.31(0.90-1.91)$ & 0.157 \\
\hline Coronary artery disease & $0.89(0.62-1.28)$ & 0.531 \\
\hline Charlson score & $0.95(0.88-1.03)$ & 0.194 \\
\hline History of PTCA with stents & $1.24(0.54-2.83)$ & 0.607 \\
\hline History of CABG & $1.11(0.88-1.40)$ & 0.364 \\
\hline ICU admission SOFA & $1.01(0.95-1.08)$ & 0.765 \\
\hline ICU admission qSOFA & $0.89(0.68-1.17)$ & 0.409 \\
\hline \multicolumn{3}{|l|}{ Etiology of AKI } \\
\hline Cardiovascular event & $0.88(0.52-1.47)$ & 0.617 \\
\hline Sepsis & $1.00(0.81-1.24)$ & 0.983 \\
\hline \multicolumn{3}{|c|}{ Clinical parameters before dialysis } \\
\hline SBP & $1.01(1.00-1.01)$ & 0.141 \\
\hline Daily UO $(\log ), \mathrm{ml}$ & $1.28(1.01-1.61)$ & 0.040 \\
\hline GCS & $1.05(0.98-1.11)$ & 0.145 \\
\hline $\mathrm{Hgb}$ & $1.09(1.00-1.18)$ & 0.043 \\
\hline $\mathrm{Na}$ & $1.02(1.00-1.04)$ & 0.045 \\
\hline K & $1.20(0.97-1.47)$ & 0.088 \\
\hline Sugar & $1.00(1.00-1.00)$ & 0.444 \\
\hline Lactate $>4.2 \mathrm{mmol} / \mathrm{L}$ & $0.07(0.01-0.50)$ & 0.009 \\
\hline SOFA & $0.97(0.88-1.07)$ & 0.528 \\
\hline \multicolumn{3}{|l|}{ qSOFA } \\
\hline 1 & $0.14(0.04-0.44)$ & 0.001 \\
\hline 2 & $0.15(0.05-0.45)$ & 0.001 \\
\hline 3 & $0.19(0.06-0.61)$ & 0.005 \\
\hline qSOFA $x$ high Lactate ${ }^{\text {II }}$ & & \\
\hline
\end{tabular}




\begin{tabular}{|l|c|c|}
\hline qSOFA=1, high v.s. low Lacate & $17.36(2.13-141.25)$ & 0.008 \\
\hline qSOFA=2, high v.s. low Lacate & $9.35(1.13-77.63)$ & 0.038 \\
\hline qSOFA=3, high v.s. low Lacate & $6.17(0.66-57.37)$ & 0.110 \\
\hline
\end{tabular}

Abbreviations: AKI, acute kidney injury; Hgb, hemoglobin; CABG, coronary artery bypass graft; FC, functional class; GCS, Glasgow coma scale; ICU, intensive care unit; MI, myocardial infarction; NYHA, New York Heart Association; PTCA, percutaneous transluminal coronary angioplasty; qSOFA, quick Sequential Organ Failure Assessment; SBP, systolic blood pressure; SCr, serum creatinine; SOFA, Sequential Organ Failure Assessment; TPN, total parental nutrition; UO, urine output

${ }^{\text {II }}$ Pre-dialysis lactate $\leqq 4.2 \mathrm{mmol}$ was defined as low lactate Pre-dialysis lactate $>4.2 \mathrm{mmol}$ was defined as high lactate 
Table 4. Incidence rate ratios and E-values of pre-dialytic qSOFA score and lactate for mortality in type $1 \mathrm{CRS}$ patients.

\begin{tabular}{|l|c|c|c|l|c|c|}
\hline Parameter & Total & Event & $\begin{array}{l}\text { Mean follow up } \\
\text { days (SD) }\end{array}$ & $\begin{array}{l}\text { Incidence } \\
\text { rate/100,000 PY }\end{array}$ & $\begin{array}{l}\text { Incidence rate ratio } \\
(95 \% \text { CI })\end{array}$ & $\begin{array}{l}\text { E-value (E-value for } \\
\text { lower limit of CI) }\end{array}$ \\
\hline qSOFA=1 & 103 & 30 & $107.13(64.74)$ & 271.89 & $0.58(0.18-2.99)$ & $2.82(1.00)$ \\
\hline qSOFA=2 & 291 & 168 & $81.66(74.17)$ & 706.98 & $1.52(0.510-7.43)$ & $2.66(1.00)$ \\
\hline qSOFA=3 & 100 & 63 & $77.61(82.33)$ & 811.75 & $1.74(0.570-8.68)$ & $3.17(1.00)$ \\
\hline Lactate & 288 & 180 & $74.56(74.90)$ & 838.30 & $1.38(1.147-1.65)$ & $2.32(1.56)$ \\
\hline
\end{tabular}




\section{Figures}

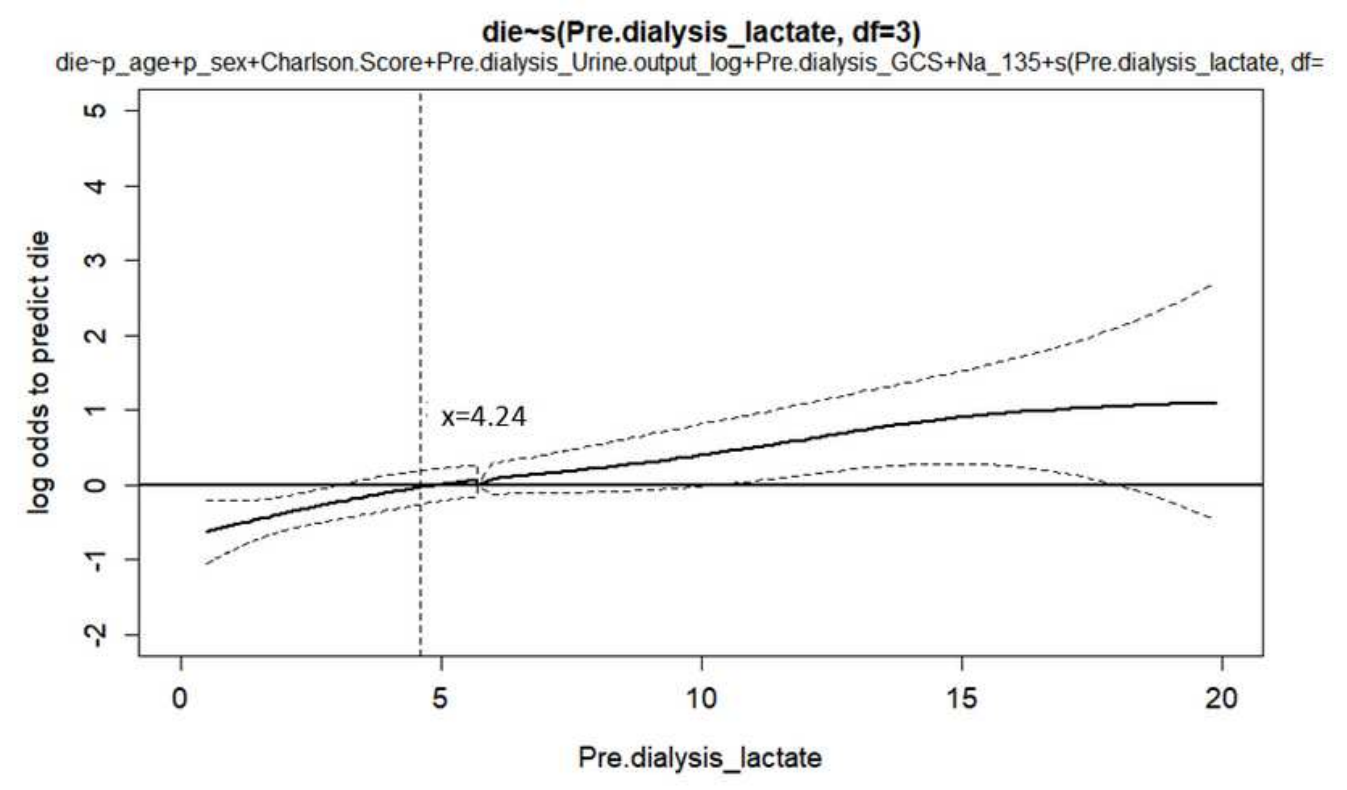

\section{Figure 1}

Generalized additive model plot for the probability of 90-day mortality against serum lactate levels at the initiation of dialysis. The generalized additive model plot was incorporated with subject-specific random effects expressed as the logarithm of the odds (logit). The probability of outcome events was constructed with lactate levels averaging zero over the range of the data, i.e. lactate $=4.24 \mathrm{ng} / \mathrm{mL}$. Abbreviations: AKI, acute kidney injury; ICU, intensive care unit; qSOFA, quick Sequential Organ Failure Assessment 9 Predialysis lactate level $\otimes 4.2 \mathrm{mmol}$ was defined as low lactate Pre-dialysis lactate level $>4.2 \mathrm{mmol}$ was defined as high lactate 


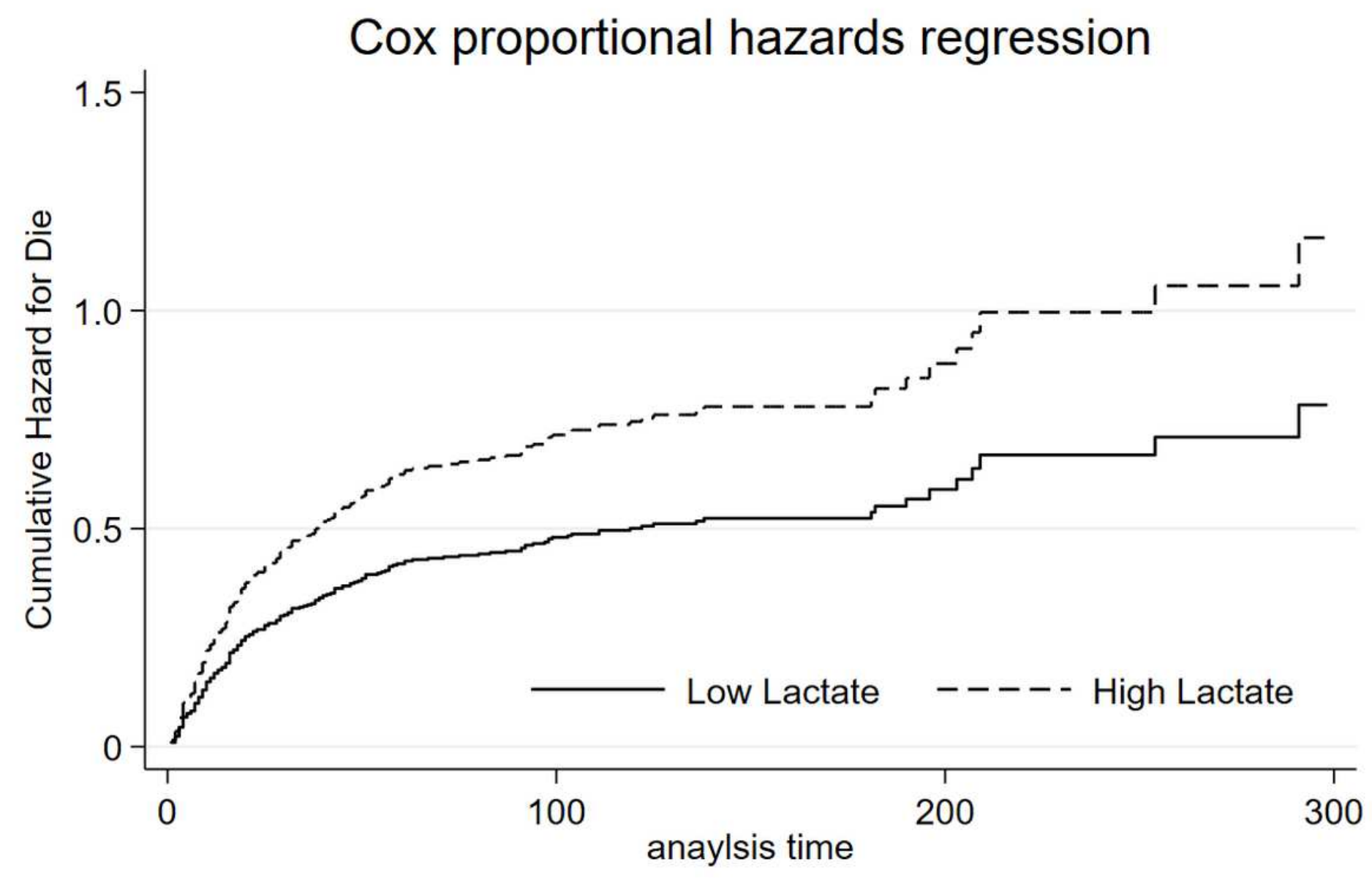

Figure 2

Cox proportional hazard plots stratified by pre-dialysis serum lactate level for assessing the probability of mortality. All relevant covariates, including characteristics, comorbidities and laboratory data at ICU admission, etiology of AKI, indication for dialysis, dialysis modality, qSOFA score, and plasma lactate level at dialysis, and some of their interactions including those listed in Table 1 were put on a selected variable list to predict the outcome of interest. 


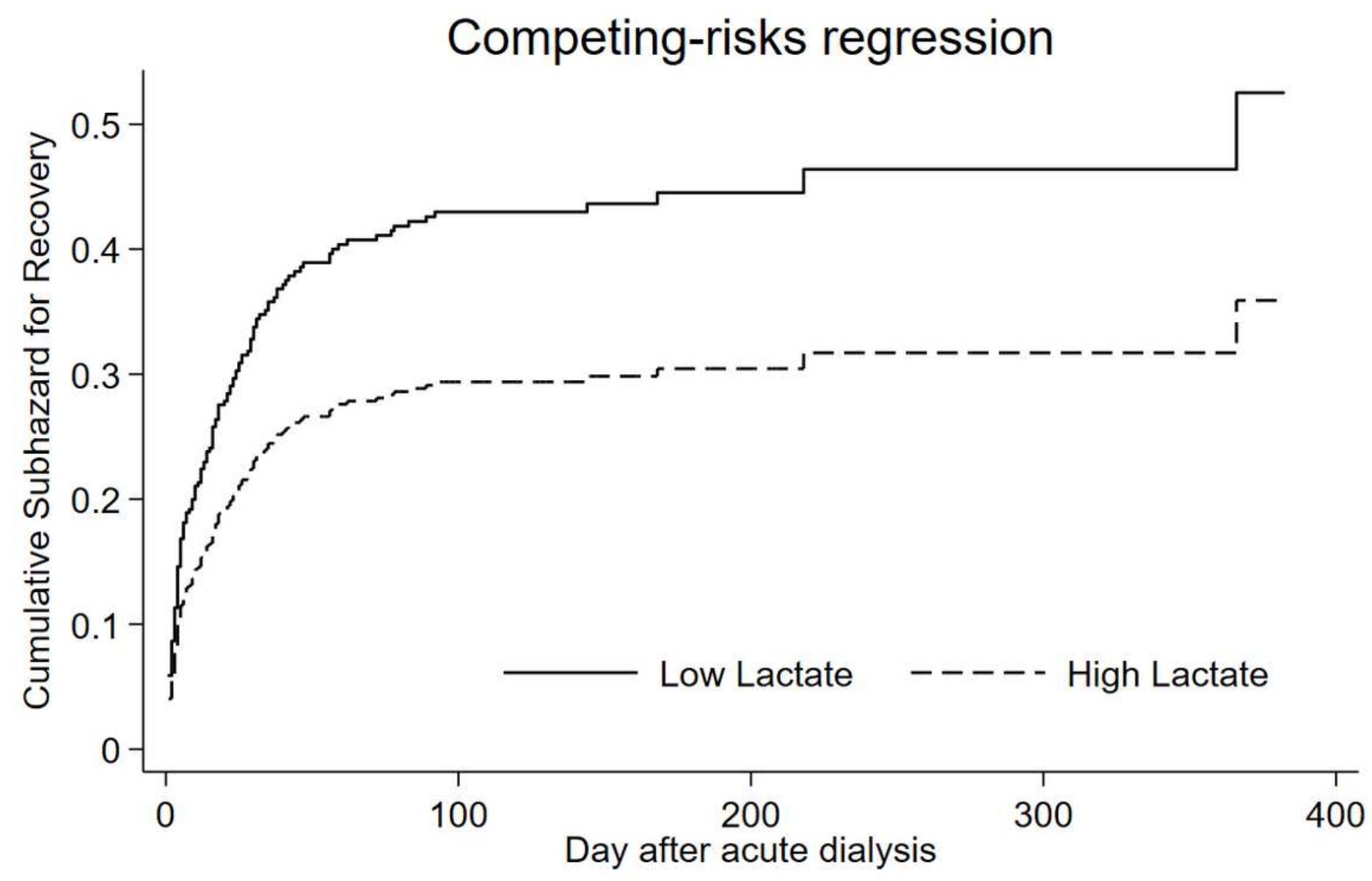

Figure 3

Cox proportional hazard plots stratified by pre-dialysis serum lactate level for assessing probability of dialysis withdraw, taking mortality as a competing risk 9 . 9 Pre-dialysis lactate level $₫ \mathbf{4 . 2} \mathrm{mmol}$ was defined as low lactate Pre-dialysis lactate level $>4.2 \mathrm{mmol}$ was defined as high lactate 


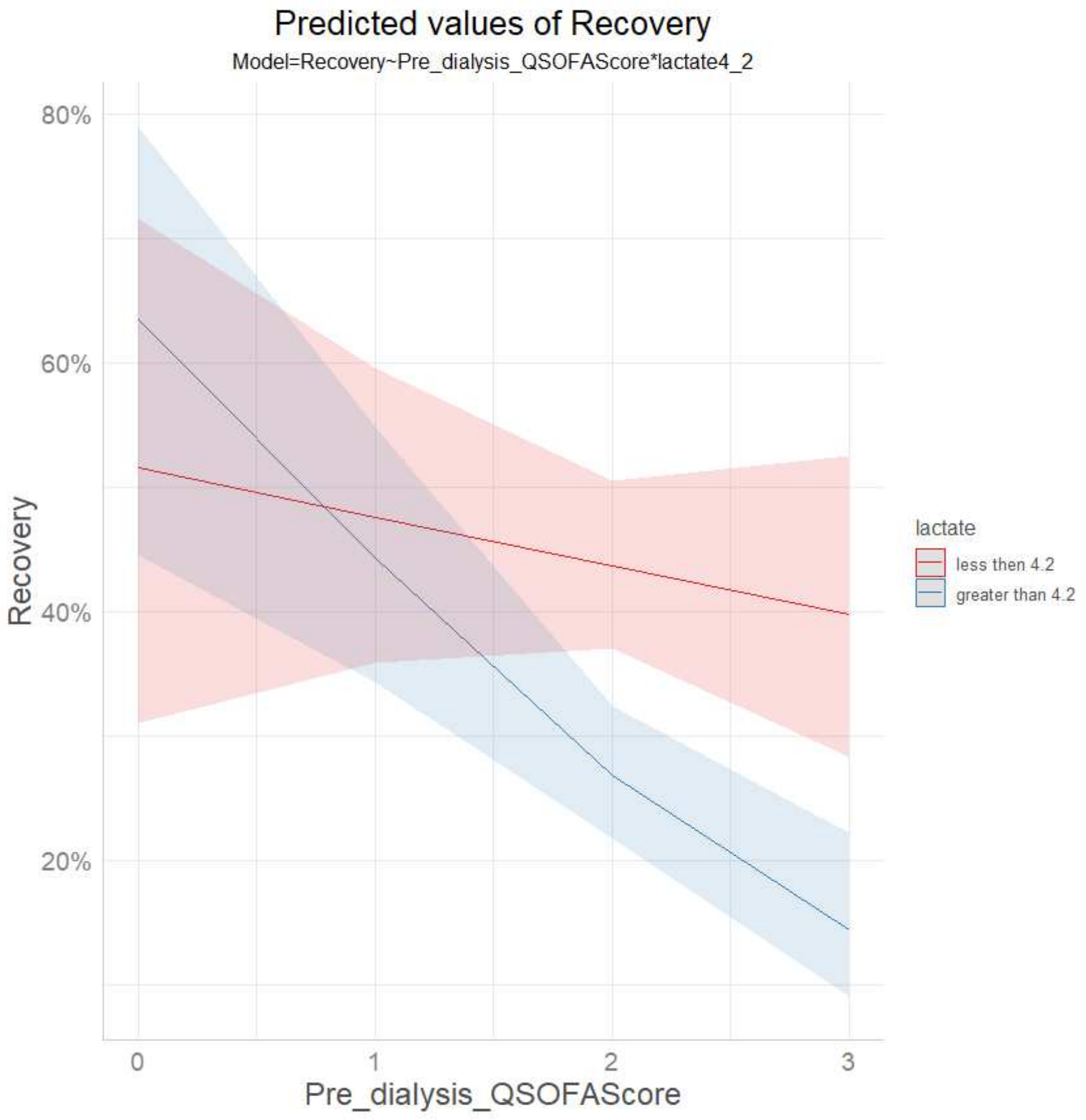

\section{Figure 4}

Marginal effects of the interaction between probability of dialysis withdrawal and predialysis qSOFA score according to a high ( $>4.2 \mathrm{mmol} / \mathrm{L})$ or low $(\varangle 4.2 \mathrm{mmol} / \mathrm{L})$ predialysis serum lactate level. The crossed blue and red lines supported that there was an interaction effect, in which an impressive interaction between the probability of dialysis withdrawal and predialysis qSOFA score was confirmed under the influence of a high and low lactate level. The graph showed that the probability of dialysis 


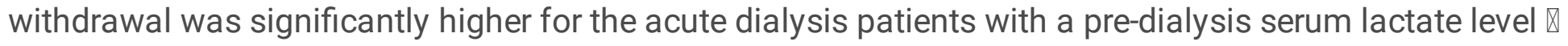
$4.2 \mathrm{mmol} / \mathrm{L}(p<0.001)$.

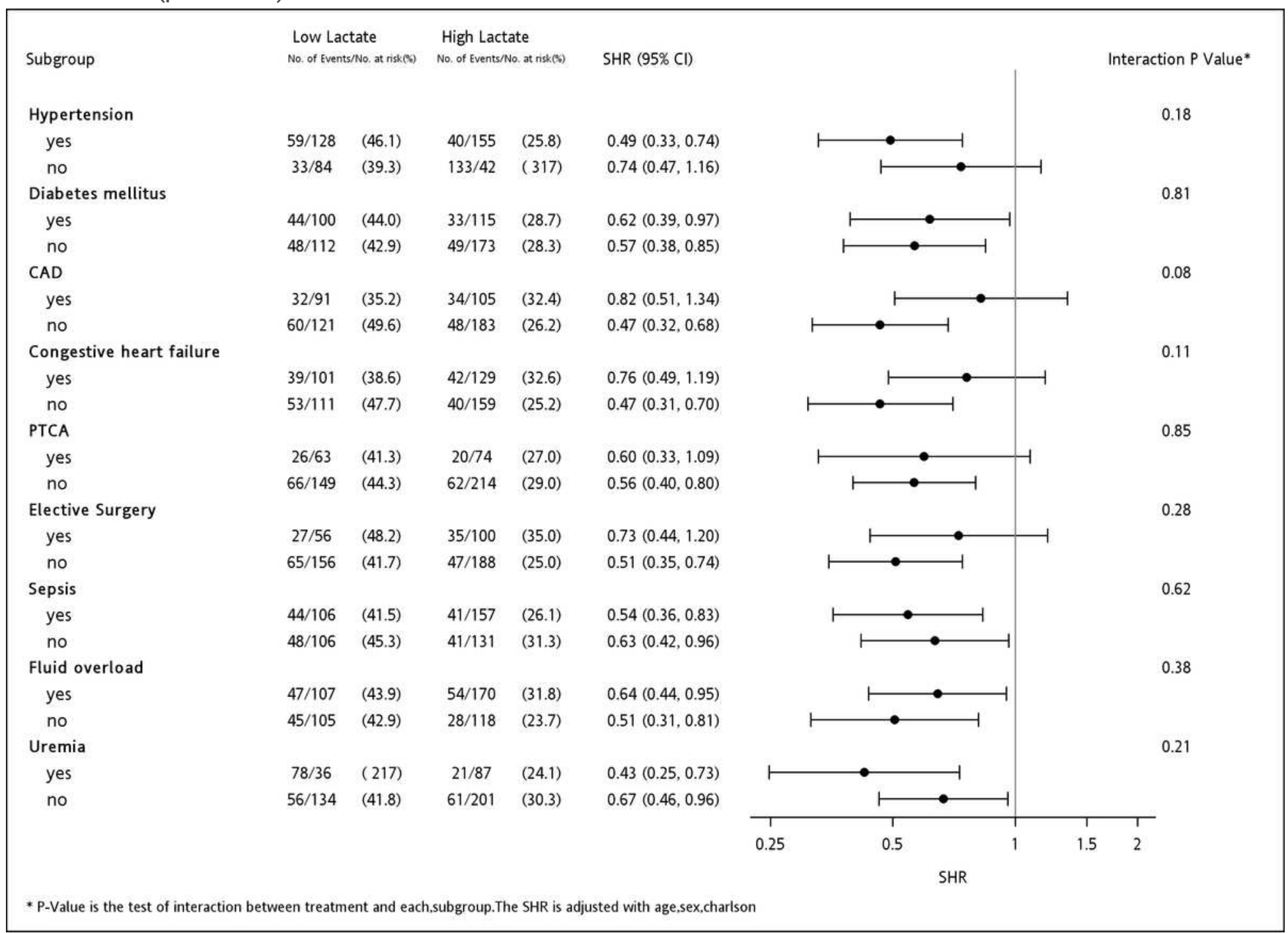

\section{Figure 5}

Forest plot depicting subgroup analysis of dialysis withdrawal compared with high and low pre-dialysis serum levels of lactate, taking mortality as a competing risk $\mathbf{9}$. Abbreviations: CAD, coronary artery disease; PTCA, percutaneous transluminal coronary angioplasty; 9 Pre-dialysis lactate level $₫ 4.2 \mathrm{mmol}$ was defined as low lactate Pre-dialysis lactate level $>4.2 \mathrm{mmol}$ was defined as high lactate

\section{Supplementary Files}

This is a list of supplementary files associated with this preprint. Click to download.

- SupplFigureTable.pdf 